OPEN ACCESS

Edited by:

Ashok Kumar,

University of Florida, USA

Reviewed by:

Gunnar P. H. Dietz,

Schwabe Pharma Deutschland,

Germany

Andrew Scholey,

Swinburne University of Technology,

Australia

*Correspondence:

Guo-Qing Zheng

gq_zheng@sohu.com

Yan Lin

linyanm@vip.sina.com

Received: 22 April 2016 Accepted: 04 November 2016 Published: 06 December 2016

Citation:

Zhang $H-F$, Huang $L-B$, Zhong $Y-B$,

Zhou Q-H, Wang $H-L$, Zheng G-Q and

Lin $Y$ (2016) An Overview of

Systematic Reviews of Ginkgo biloba

Extracts for Mild Cognitive Impairment and Dementia.

Front. Aging Neurosci. 8:276. doi: 10.3389/fnagi.2016.00276

\section{An Overview of Systematic Reviews of Ginkgo biloba Extracts for Mild Cognitive Impairment and Dementia}

\author{
Hong-Feng Zhang ${ }^{1}$, Li-Bo Huang ${ }^{1}$, Yan-Biao Zhong ${ }^{2}$, Qi-Hui Zhou ${ }^{1}$, Hui-Lin Wang ${ }^{1}$, \\ Guo-Qing Zheng ${ }^{1 *}$ and Yan Lin ${ }^{1 *}$
}

${ }^{1}$ Department of Neurology, The Second Affiliated Hospital and Yuying Children's Hospital of Wenzhou Medical University, Wenzhou, China, ${ }^{2}$ Department of Rehabilitation, The Second Affiliated Hospital and Yuying Children's Hospital of Wenzhou Medical University, Wenzhou, China

Ginkgo biloba extracts (GBEs) have been recommended to improve cognitive function and to prevent cognitive decline, but earlier evidence was inconclusive. Here, we evaluated all systematic reviews of GBEs for prevention of cognitive decline, and intervention of mild cognitive impairment $(\mathrm{MCl})$ and dementia. Six databases from their inception to September 2015 were searched. Ten systematic reviews were identified, including reviews about Alzheimer's disease $(n=3)$, about vascular dementia $(n=1)$, about both Alzheimer's disease and vascular dementia $(n=2)$, about Alzheimer's disease, vascular dementia and mixed dementia $(n=3)$, and a review about $\mathrm{MCl}(n=1)$. Based on the overview quality assessment questionnaire, eight studies were scored with at least 5 points, while the other two scored 4 points and 3 points, respectively. Medication with GBEs showed improvement in cognition, neuropsychiatric symptoms, and daily activities, and the effect was dose-dependent. Efficacy was convincingly demonstrated only when high daily dose $(240 \mathrm{mg})$ was applied. Compared with placebo, overall adverse events and serious adverse events were at the same level as placebo, with less adverse events in favor of GBE in the subgroup of Alzheimer's disease patients, and fewer incidences in vertigo, tinnitus, angina pectoris, and headache. In conclusion, there is clear evidence to support the efficacy of $\mathrm{GBEs}$ for $\mathrm{MCl}$ and dementia, whereas the question on efficacy to prevent cognitive decline is still open. In addition, GBEs seem to be generally safe.

Keywords: Ginkgo biloba extracts, dementia, Alzheimer's disease, vascular dementia, mild cognitive impairment

\section{INTRODUCTION}

Cognitive decline is a major social problem of public health (Hugo and Ganguli, 2014; Hill et al., 2015). Cognitive decline includes mild cognitive impairment (MCI) and dementia (Howieson, 2016; Jørgensen et al., 2016; Mormino and Papp, 2016; Ströhle and Rapp, 2016; Thomas, 2016). $\mathrm{MCI}$ is characterized by a slight but noticeable decline in cognitive function, which is regarded as a symptomatic stage before progression to dementia (Budson and Solomon, 2012; Thung et al., 2015; Fernández-Blázquez et al., 2016; Petersen, 2016). MCI is further categorized into MCI due to Alzheimer's disease (AD) and MCI due to other causes. Dementia is a chronic acquired progressive mental retardation syndrome (Damiani et al., 2014; Noel-Storr et al., 2014; Ihl et al., 2015; Mitchell, 2015; Ngo and Holroyd-Leduc, 2015; Wang et al., 2016). The causes include AD, vascular dementia 
(VD), and mixed dementia (Montine et al., 2014; Altamura et al., 2016). AD is the most common neurodegenerative disease, and generally begins with mild memory problems, progressing to the development of multiple cognitive and functional impairment within a few years (Brooker et al., 2014; Dubois et al., 2014; Aygün and Güngör, 2015; Apostolova, 2016; Wood, 2016). VD is a severe cognitive dysfunction syndrome caused by ischemic stroke, hemorrhagic stroke, and cerebral vascular disease with low cerebral perfusion which leads to the impairment of memory, cognition, and behavior (Tsivgoulis et al., 2014; O'Brien and Thomas, 2015). Mixed dementia means that both $\mathrm{AD}$ and $\mathrm{VD}$ occur in one patient (Moore et al., 2014; Bogolepova, 2015; Kim et al., 2016). Currently, more than 46 million people live with dementia worldwide, and the number is estimated to increase to 131.5 million by 2050 (Prince et al., 2015). Dementia also has a huge economic impact. The total worldwide cost was US\$ 818 billion in 2015, US\$ 604 billion in 2010, and is estimated to be 1 trillion in 2018 (Prince et al., 2015).

The treatment for dementia and MCI is still symptomatic (Fitzpatrick-Lewis et al., 2015). Up to now, no diseasemodifying therapy has been available (Kennedy and Sud, 2014). Cholinesterase inhibitors increase the concentration of neurotransmitter in the brain and improve memory (Rockwood et al., 2013; Yáñez and Viña, 2013; Chen et al., 2016); NMDA receptor antagonists reduce neurotoxicity through inhibiting excitatory amino acid receptors (Newport et al., 2015; Schmidt et al., 2015); other drugs such as Serotonergic agents, Dopamine blocking agents, Benzodiazepines alleviate specific other symptoms (Schneider et al., 2014; Deardorff et al., 2015). Thus, there are multiple alternative strategies with moderate efficacy for some patients for a limited amount of time.

Ginkgo biloba extracts (GBEs) are widely used for various kinds of disorders, including cognitive dysfunctions, headache, tinnitus, vertigo, inattention, mood disturbances, cardiovascular diseases, and coronary heart disease (DeFeudis and Drieu, 2000). Ginkgo biloba contains flavonoids, terpene lactones, and ginkgolic acids (Oken et al., 1998). GBEs have been demonstrated to have antioxidative activities, to increase tolerance to hypoxia, and to improve blood rheology by increasing the flexibility of cellular blood components, thus enhancing microcirculation; affecting neurotransmitter levels; enhancing neuroplasticity; prevention of brain edema; and neuroprotection (DeFeudis and Drieu, 2000; Tchantchou et al., 2007, 2009; Fehske et al., 2009; Yoshitake et al., 2010; Altamura et al., 2016). GBEs have been widely used in the treatment of dementia for decades now (Weitbrecht and Jansen, 1986; Oken et al., 1998). Some systematic reviews have been conducted to assess GBEs in prevention and treatment of MCI and dementia (Janssen et al., 2010; Wang et al., 2010; Weinmann et al., 2010; Yang et al., 2011, 2014, 2016; Hu et al., 2013; Jiang et al., 2013; Gauthier and Schlaefke, 2014; Tan et al., 2015). To provide an overview over the large amount of available data, we here provide a systematic review of those systematic reviews.

\section{METHODS}

\section{Search Strategy}

Systematic reviews and meta-analyses of GBEs for prevention, $\mathrm{MCI}$, and dementia were searched in six databases from their inception to September 2015. The databases included Chinese biological medical literature database, Web of science, Pubmed, Chinese Wanfang data, Chinese VIP information, and Chinese national knowledge infrastructure. The search terms used were ["Ginko biloba" OR ("Ginkgo biloba" AND extract) OR "EGb 761"] AND ("cognitive function decline" OR "mild cognitive impairment" OR "mixed dementia" OR "Alzheimer's disease" OR "vascular dementia”) AND (“systematic review" OR "metaanalysis") in English and Chinese. The reference lists of all relevant articles were searched for additional studies.

\section{Inclusion Criteria and Exclusion Criteria}

Studies that met all of the following criteria were included: (i) the articles were systematic reviews and/or meta-analysis; (ii) the publications reported the use of GBEs for prevention of cognitive function decline, or for intervention of MCI, and dementia, including $\mathrm{AD}, \mathrm{VD}$, or mixed dementia; (iii) studies were limited to humans.

Studies were excluded if they were any of the following: (i) systematic reviews evaluating other Chinese herbal medicine or mixed treatments; (ii) non-systematic reviews, comments, and overviews.

\section{Study Selection and Data Collection}

All articles were read by two independent reviewers (YBZ, HFZ) and data from the articles were extracted and validated according to predefined criteria. The following details of the article were extracted from the included studies: (1) first author's name and the publication year; (2) the types of diseases, number of primary studies, and quality of primary studies; (3) the conclusions and meta analyses of primary studies, and the nature of the extracts of Ginkgo biloba; (4) the overall scores and each items on OQAQ; (5) quality assessment, quality of randomized controlled trials, inclusion criteria and exclusion criteria of primary studies. Data for meta-analyses were based on the reported summary statistics for each study. Disagreements regarding inclusion and quality were settled through discussion or consultation with the corresponding author.

\section{Quality Assessment}

Two investigators independently (YBZ and HFZ) assessed the methodological quality of all included systematic reviews by using the overview quality assessment questionnaire (OQAQ) (Al Faleh and Al-Omran, 2009). The OQAQ is composed of 10 questions, graded on a 7-point scale. Questions 1-9, which were answered with "adequate," "inadequate," or "not mentioned," addressed the five methodological aspects of systematic reviews, including search strategy, study selection, validity assessment, data analysis, and conclusions. Only the answer "adequate" receives a positive score, while "inadequate" or "not mentioned" scored 0 point. Each of questions $1-4$ and 9 values 1 point, while each of questions $5-8$ is 0.5 points. The final question 10 is to conclude the whole points of the scientific quality of these reviews 


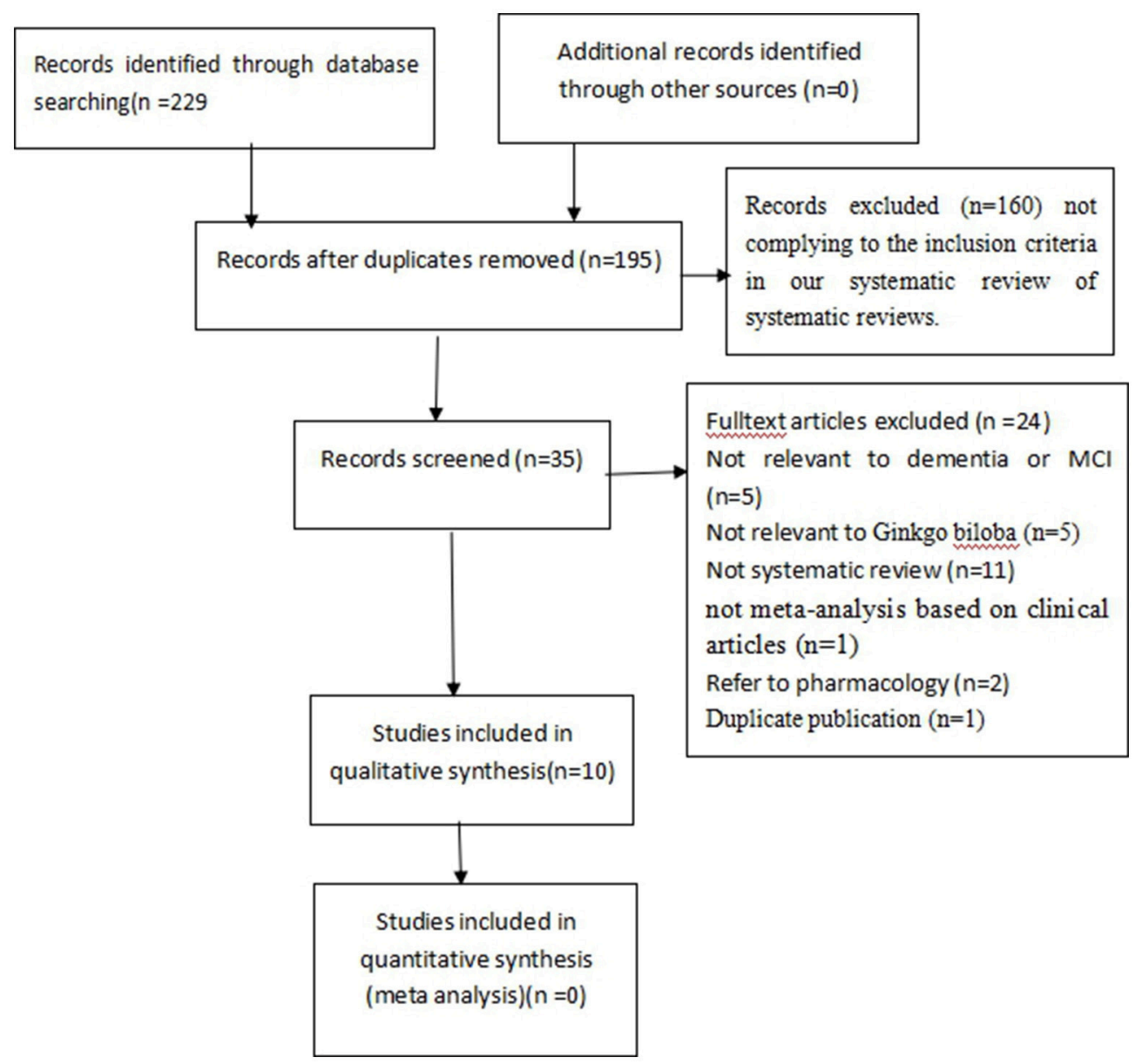

FIGURE 1 | Flow Diagram of included systematic reviews.

we rate. A score of three or less was regarded as indicative of extensive or major flaws and a score of 5 or more as suggesting minor or minimal flaws.

\section{RESULTS}

\section{Description of the Screening Process}

The search strategy yielded 229 potentially relevant hits. After removal of duplicates, 195 records remained. Through screening titles and abstracts, we excluded 160 papers. In the remaining 35 papers, 25 papers were excluded with at least one of following reasons: (1) not relevant to dementia or MCI $(n=5)$; (2) not relevant to GBEs $(n=5)$; (3) not a systematic review $(n=11)$; (4) not a meta-analysis based on clinical articles $(n=1)$; $(5)$ animal studies $(n=2)$; (6) duplicated publication $(n=1)$. Ultimately, 10 eligible studies were selected (Figure 1).

\section{Study Characteristics}

The 10 systematic reviews included were published between 2010 and 2015. Among them, 9 systematic reviews were published in English and 1 systematic review in Chinese (Jiang et al., 2013).
7 first authors of the 10 systematic reviews were from China (Wang et al., 2010; Yang et al., 2011, 2014, 2016; Hu et al., 2013; Jiang et al., 2013; Tan et al., 2015), 1 from Canada (Gauthier and Schlaefke, 2014), and 2 from Germany (Janssen et al., 2010; Weinmann et al., 2010). The 10 systematic reviews included reviews about Alzheimer's disease $(n=3)$, about vascular dementia $(n=1)$, about both Alzheimer's disease and vascular dementia $(n=2)$, about Alzheimer's disease, vascular dementia and mixed dementia $(n=3)$, and a review about MCI $(n=$ 1) (Table 1). The inclusion criteria, the quality of randomized controlled trials, exclusion criteria, and the quality assessment in studies are presented in Table 2. Four systematic reviews (Janssen et al., 2010; Hu et al., 2013; Gauthier and Schlaefke, 2014; Yang et al., 2014) assessed methodological quality according to the Jadad score. Five systematic reviews (Wang et al., 2010; Yang et al., 2011, 2016; Jiang et al., 2013; Tan et al., 2015) used the Cochrane Collaboration guidelines to evaluate methodological quality. The remaining systematic review (Weinmann et al., 2010) assessed methodological quality according to the modified Cochrane Collaboration guidelines. The randomized controlled trials (RCTs) compared GBEs with either placebo or Western 
conventional medicine (WCM) in patients with MCI, VD, AD, and/or mixed dementia. The quality of randomized controlled trials in 7 studies (Wang et al., 2010; Weinmann et al., 2010; Jiang et al., 2013; Gauthier and Schlaefke, 2014; Yang et al., 2014, 2016; Tan et al., 2015) are good, while moderate in 2 studies (Janssen et al., 2010; Yang et al., 2011) and poor in 1 study (Hu et al., 2013). Seven systematic reviews (Wang et al., 2010; Weinmann et al., 2010; Yang et al., 2011, 2016; Jiang et al., 2013; Gauthier and Schlaefke, 2014; Tan et al., 2015) reported inclusion criteria and exclusion criteria, while three (Janssen et al., 2010; Hu et al., 2013; Yang et al., 2014) reported inclusion criteria only. Key data of included systematic reviews are summarized in Tables 1, 2.

\section{Assessing the Quality of Systematic Reviews}

According to OQAQ scores, the quality of these systematic reviews varied. Nine studies included (Janssen et al., 2010; Wang et al., 2010; Weinmann et al., 2010; Yang et al., 2011, 2014, 2016; Jiang et al., 2013; Gauthier and Schlaefke, 2014; Tan et al., 2015; Table 3) were considered to have only minor or minimal flaws, i.e., scored with at least 5 points on the OQAQ. One systematic review was seriously flawed and scored 3 points only (Hu et al., 2013; Table 3). Although the review by Yang et al. (2016) received an OQAQ score of 6 , this study is not useful because in the analysis it seems that no careful distinction between MCI and dementia was performed and because some important trials reporting on $\mathrm{AD}$ subgroups are missing (e.g., Kanowski and Hoerr, 2003; Napryeyenko et al., 2009).

\section{Effectiveness Prevention}

Only two systematic reviews mentioned prevention of cognitive function decline and the results were not significant. In one review (Yang et al., 2014), the preventative effect of AD were studied in two studies (DeKosky et al., 2008; Vellas et al., 2012), while in the other review (Yang et al., 2016), only one study was included (Vellas et al., 2012). However, in the study by DeKosky et al. (2008) an extremely low compliance was a serious issue; in both trials the overall conversion rate to dementia was too low to draw conclusions on the efficacy, as both studies were highly underpowered. In addition, the study by Vellas et al. (2012) was inconclusive. Not only was there a significantly lower rate of progression to $\mathrm{AD}$ in the prospectively specified subgroup of subjects who were on drug for at least 4 years, there was also a problem with the pre-specified statistical test which was chosen assuming proportional hazards. When this assumption turned out wrong, because hazards were found to increase during the study period, a post-hoc analysis was performed by Scherrer et al. (2015) which demonstrated a significantly lower rate of progression to $\mathrm{AD}$ in the Extract Ginkgo biloba number 761 (EGb 761) ${ }^{\circledR}$-treated subjects when an appropriate statistical test was applied that accounts for the non-proportional hazards. Hence, the question whether GBE can prevent dementia or cognitive remains to open and needs to be examined in the future.

\section{$\mathrm{MCl}$}

(1) Activities of daily living (ADL): There were no significant difference between GBEs plus WCM and WCM alone (Yang et al., 2016). (2) Cognition function: Both GBEs alone and GBEs plus WCM were more effective than WCM (Yang et al., 2016).

\section{Dementia}

(1) Global clinical assessment: Two systematic reviews (Wang et al., 2010; Gauthier and Schlaefke, 2014) indicated that EGb 761 was better than placebo. Gauthier and Schlaefke (2014) further demonstrated that EGb 761 was dose-dependently effective, with and high daily dose $(240 \mathrm{mg}$ ) of EGb 761 with better efficacy compared to low dose (120 mg). (2) ADL: One systematic review (Tan et al., 2015) indicated that GBEs could improve ADL in dementia patients, but another one (Weinmann et al., 2010) did not show such effect, because that analysis could not include several of the more recent and successful trials. Jiang et al. (2013) specifically demonstrated that EGb 761 could improve ADL in relatively young (age $<75$ years) patients but not in those older than 75 . However, only two trials were included in the age group above 75 years. When 4 trials were included in the analysis of old patients, a clear beneficial effect of EGb 761 could be demonstrated by Kasper (2015). Moreover, Jiang et al. (2013) inappropriately included the trials by van Dongen et al. (2003) and McCarney et al. (2008). van Dongen et al. (2003) included a mixed population of subjects with aging-associated memory impairment (AAMI) and dementia. Most patients had AAMI, i.e., they had-by definition-no impairment of ADL and therefore they could hardly improve significantly in ADL. In the study by McCarney et al. (2008) substantial proportions of the Ginkgo and placebo groups (about one third) were on cholinesterase inhibitors. Effective treatment with cholinesterase inhibitors may decrease the chance and the extent of further improvement by GBE. Moreover, 25\% of the patients in the Ginkgo group, but only $10 \%$ of the patients in the placebo group had been treated with Ginkgo before enrollment. Those treated with GBE before are certainly less likely to show marked further improvement during the study. Furthermore, the study was seriously underpowered due to recruitment problems and due to the split of both groups to sub-groups with minimal and extensive follow-up, variances were particularly large, which further decreased statistical power. The inappropriate choice of trials for inclusion and the exclusion was seriously flawed and trials that enrolled different patients are essential for a review to mislead to the correct conclusion with regard to efficacy in a specific disorder. Two systematic reviews (Jiang et al., 2013; Gauthier and Schlaefke, 2014) demonstrated that the effects of high daily dose $(240 \mathrm{mg})$ on ADL were more significant than that of placebo. Although one systematic review (Gauthier and Schlaefke, 2014) indicated that relatively low daily dose (160 or $120 \mathrm{mg}$ ) of GBEs could improve ADL of dementia patients, another one (Jiang et al., 2013) had opposite results. (3) Cognition function: Two systematic reviews (Weinmann et al., 2010; Tan et al., 2015) indicated that GBEs could improve cognition function in dementia patients. Furthermore, both two systematic reviews (Jiang et al., 2013; Gauthier and Schlaefke, 2014) indicated that the effects on cognition function were 


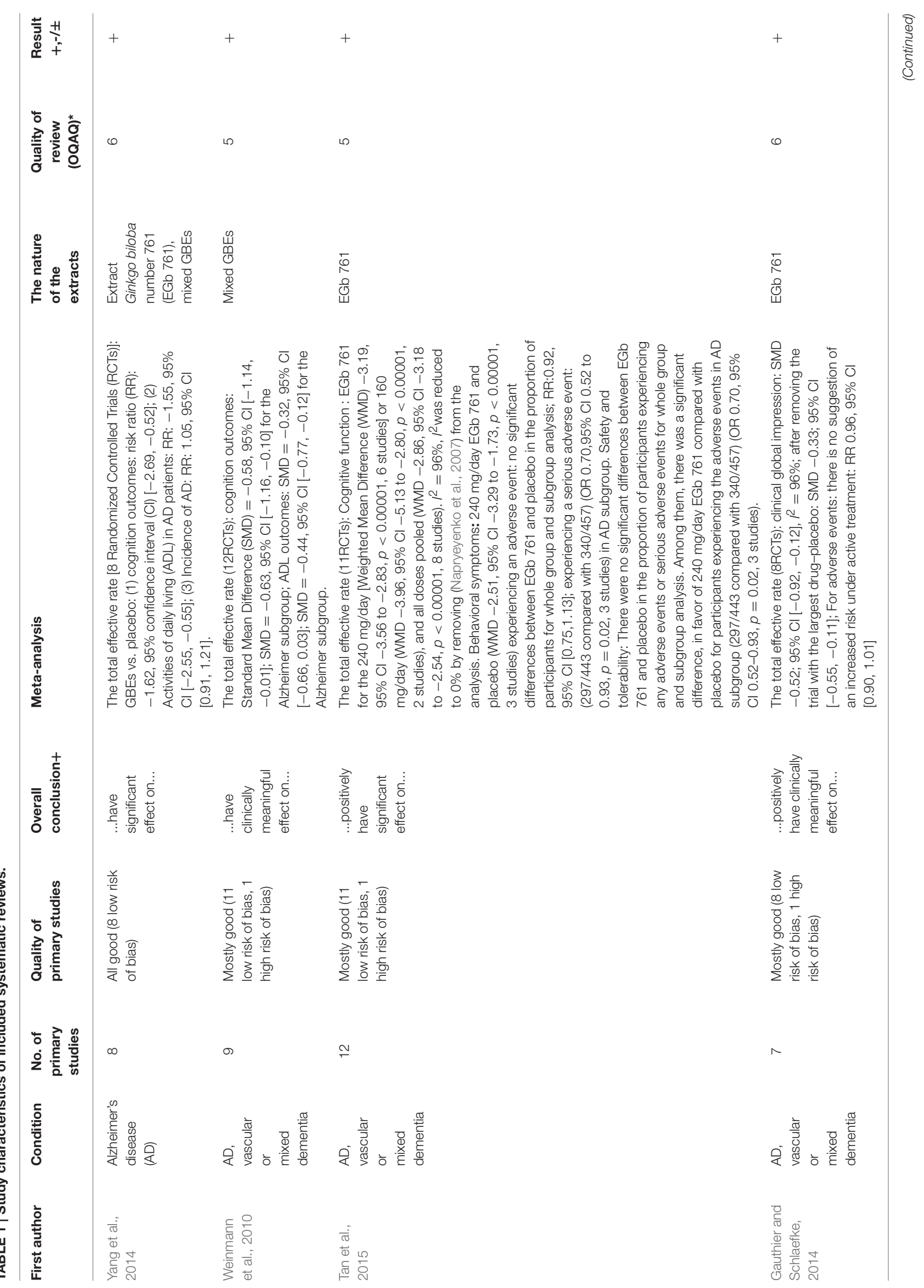




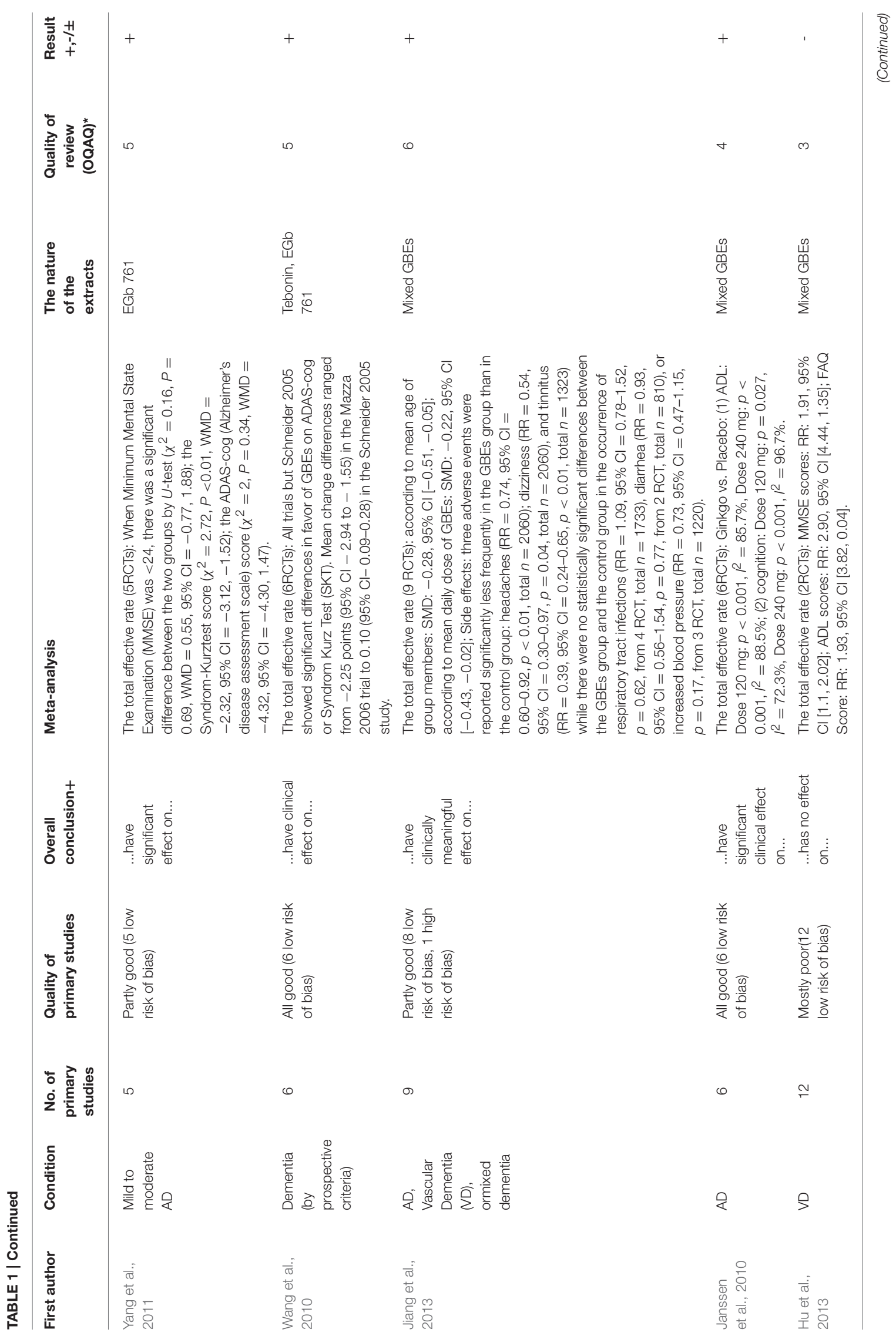



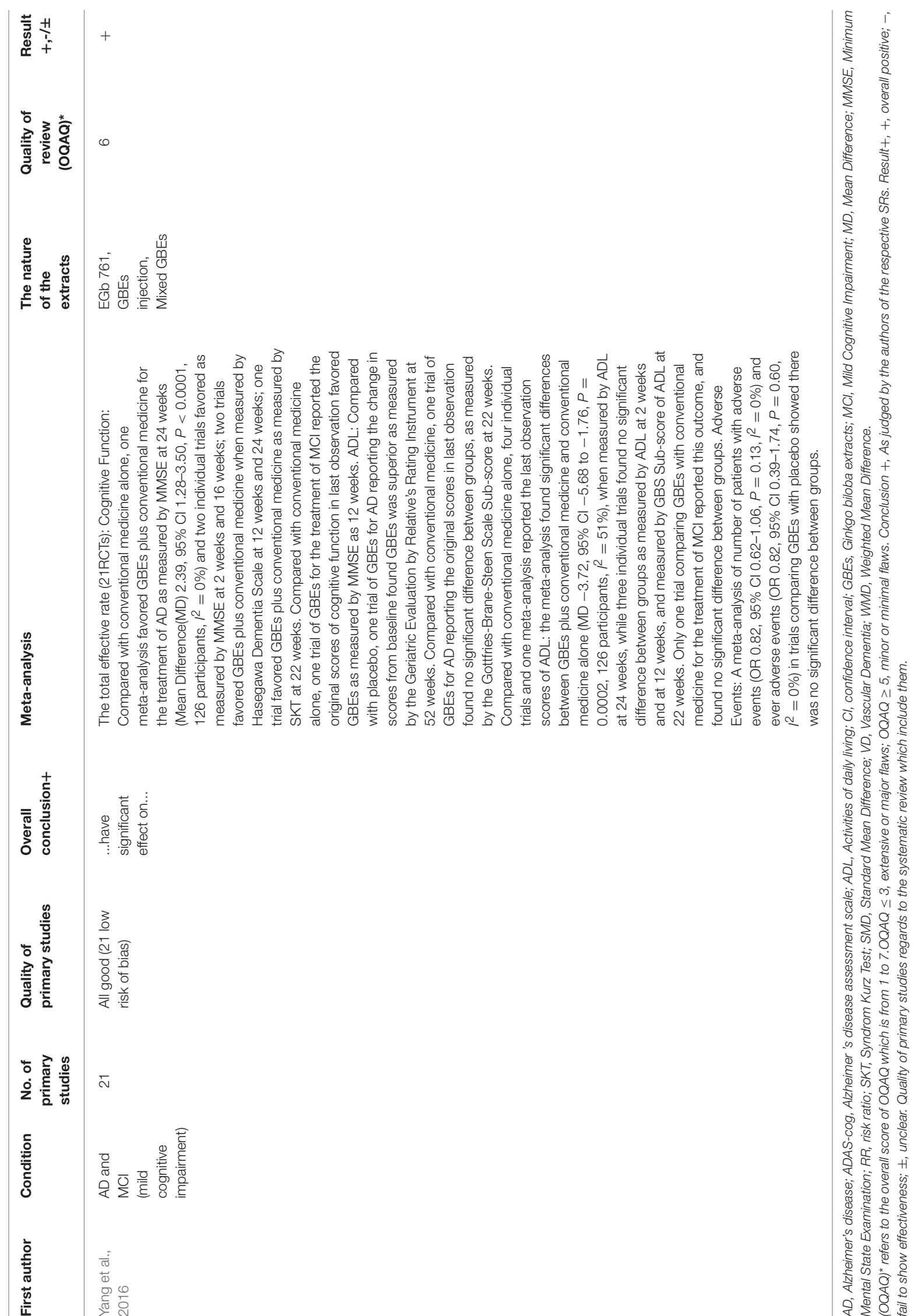


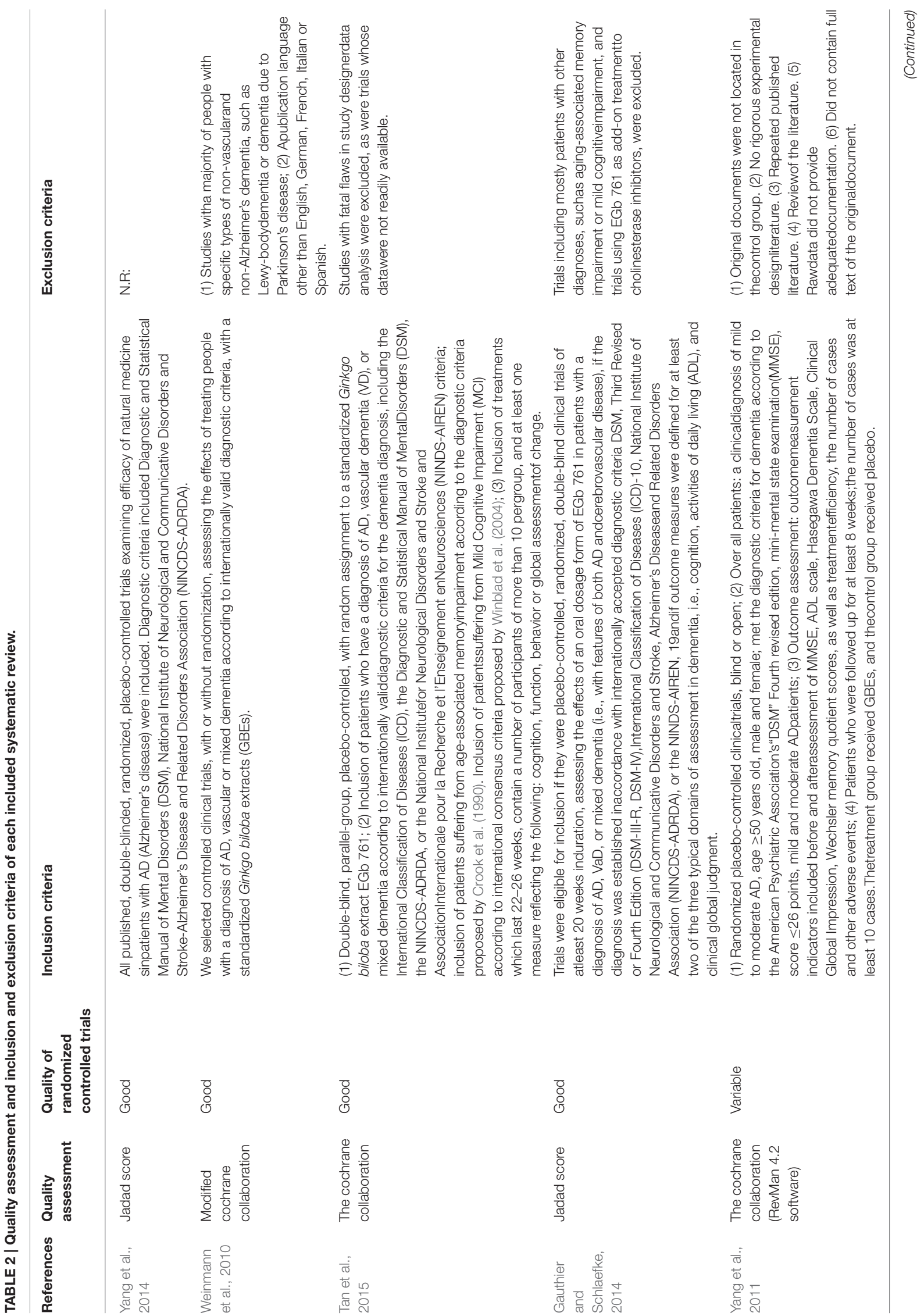




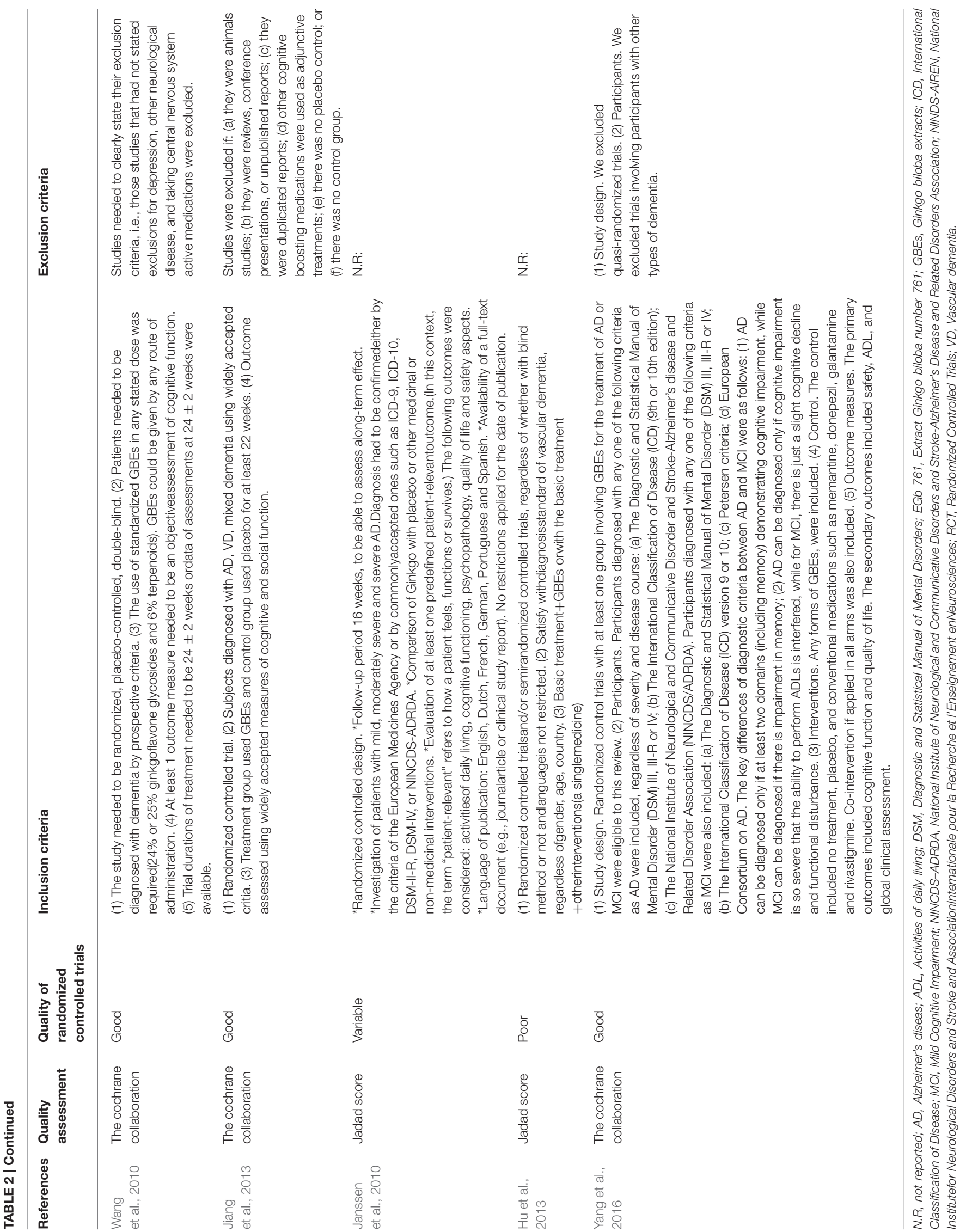




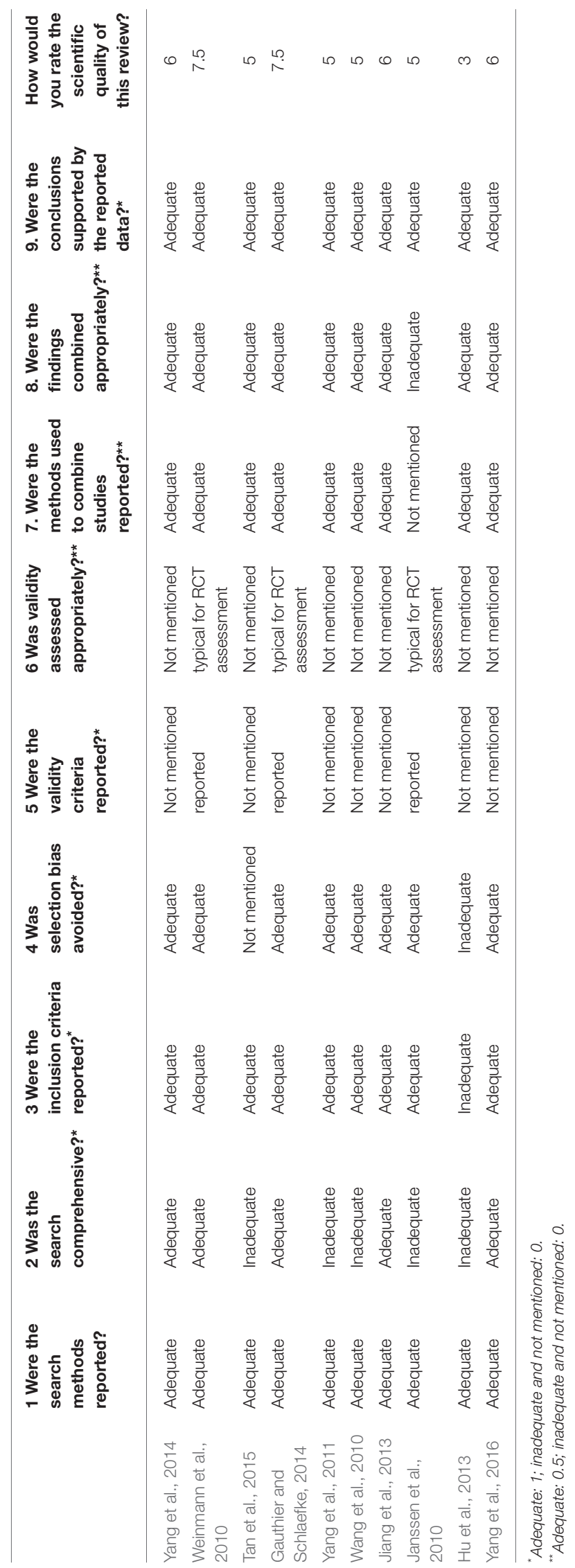

dose-dependent and relatively high daily dose $(240 \mathrm{mg})$ of GBEs could improve cognition function in dementia patients. Jiang et al. (2013) specifically indicated that EGb 761 could improve cognition function of relatively young (age $<75$ years) people but not in those older than 75. However, only two trials were included in the age group above 75 years. When four trials were included in the analysis of old patients, a clear beneficial effect of EGb 761 could be demonstrated by Kasper (2015). (4) Neuropsychiatric and behavioral symptoms (NPS): GBEs improve neuropsychiatric symptoms, including depression (Weinmann et al., 2010; Jiang et al., 2013; Gauthier and Schlaefke, 2014; Tan et al., 2015), there was a significant difference in favor of $240 \mathrm{mg}$ /day EGb761 $(p<0.00001)$ when assessing efficacy regarding improvement of neuropsychiatric symptoms (Tan et al., 2015).

\section{AD}

(1) Global clinical assessment: Two systematic reviews (Gauthier and Schlaefke, 2014; Yang et al., 2016) indicated that the effectiveness of EGb 761 was better than that of placebo. Yang et al. (2016) indicated that there was no significant difference comparing EGb 761 with WCM; however, this study is not useful because of its shortcomings explained above. (2) ADL: Four systematic reviews (Janssen et al., 2010; Weinmann et al., 2010; Yang et al., 2014; Tan et al., 2015) indicated that GBEs were effective for improving ADL. The effects on ADL were dosedependent and relatively high daily dose $(240 \mathrm{mg})$ of GBEs were more effective than placebo (Janssen et al., 2010). Yang et al. (2016) indicated that the effectiveness of GBEs was better than that of placebo but not WCM. However, due to the serious concerns regarding the review by Yang et al. (2016) mentioned above, such conclusions should be taken with a grain of salt. An additional mistake in the review by Yang et al. (2016) is that in the study by Maurer et al. (1997), there was no significant difference in the Syndrom Kurz Test (SKT) favoring EGb $761^{\circledR}$. Actually, there was a significant difference in the SKT and there was a non-significant difference in the Alzheimer's disease assessment scale-cognitive subscale (ADAS-cog), both in favor of EGb $761^{\circledR}$. The size of the change in the ADAS-cog was as expected from the size of the change in the SKT (both correlate very well), but due to the variance this difference was not significant for the ADAS-cog in the very small number of patients. In the study by Mazza et al. (2006), there also was a significant difference in favor of active treatment in the SKT, but not in the MMSE. The MMSE is a screening test for cognitive impairment and dementia and has never been validated as an outcome measure for treatment trials in dementia. Actually, in the study by Mazza et al. (2006) the difference in MMSE changes between donepezil and placebo was not significant either. These issues demonstrate again that assessing the quality of a review always involves a careful evaluation of the quality of the single trials and their correct representation in the review. (3) Cognition function: Two systematic reviews (Weinmann et al., 2010; Tan et al., 2015) indicated that GBEs improve cognition function in AD patients. (4) NPS: In an earlier analysis that could not yet take into account the most recent clinical trials, no significant effect on neuropsychiatric and behavioral symptoms could be 
demonstrated (Weinmann et al., 2010). However, in a more recent review, a significant effect of EGb 761 on neuropsychiatric symptoms could be demonstrated (Tan et al., 2015). (5) Quality of life: Yang et al. (2016) indicated that GBEs for AD was superior to placebo as measured by the quality of life questionnaire for person with dementia (DEMQOL)-proxy quality of life scale. Janssen et al. (2010) indicated that two trials available in that domain at the time that review was generated showed contradictory results. VD (Hu et al., 2013): (1) ADL: GBEs were as effective for improving $\mathrm{ADL}$ of $\mathrm{VD}$ patients compared with WCMs such as donepezil, gamma aminobutyric acid (GABA)ergic drugs or other therapies. (2) Cognition function: GBEs could improve cognition function in VD patients according to Minimum Mental State Examination (MMSE) scores.

\section{Adverse Events}

Eight systematic reviews (Janssen et al., 2010; Weinmann et al., 2010; Yang et al., 2011, 2016; Hu et al., 2013; Jiang et al., 2013; Gauthier and Schlaefke, 2014; Tan et al., 2015) examined adverse effects, while the other two (Wang et al., 2010; Yang et al., 2014) did not. Yang et al. (2011) reported that one patient had hypersensitivity. Weinmann et al. (2010) concluded that there was no difference between the GBEs group regarding adverse events and the placebo group. Janssen et al. (2010) found that, although the number of patients that discontinued the trial was higher compared to the placebo group, no evidence for a harmful effect of GBEs was observed. Interestingly, GBEs potentially reduce adverse events. Jiang et al. (2013) reported that headache $(\mathrm{RR}=0.74,95 \% \mathrm{CI}=0.60-0.92, p<0.01$, total $n=2060)$; dizziness $(\mathrm{RR}=0.54,95 \% \mathrm{CI}=0.30-0.97, p=0.04$, total $n=$ 2060), and tinnitus ( $\mathrm{RR}=0.39,95 \% \mathrm{CI}=0.24-0.65, p<0.01$, total $n=1323$ ) were significantly less frequent in the GBEs group than in the control group. There were no significant differences in the occurrence of respiratory tract infections $(\mathrm{RR}=1.09,95 \%$ $\mathrm{CI}=0.78-1.52, p=0.62$, from $4 \mathrm{RCT}$, total $n=1733$ ), diarrhea $(\mathrm{RR}=0.93,95 \% \mathrm{CI}=0.56-1.54, p=0.77$, from $2 \mathrm{RCT}$, total $n=810)$, or blood pressure elevation $(\mathrm{RR}=0.73,95 \% \mathrm{CI}=0.47-$ $1.15, p=0.17$, from 3 RCT, total $n=1220$ ) between the GBEs group and the control group. Tan et al. (2015) indicated that occurrence of dizziness, tinnitus, headache and angina pectoris in the GBEs group was lower compared to the placebo group. Gauthier and Schlaefke (2014) indicated that GBEs have no increased risk for headache, dizziness, hypertension, tinnitus, angina pectoris or respiratory tract infection. Yang et al. (2016) indicated that there was no significant difference in nausea, dizziness, headache, blood pressure elevation, respiratory tract infection, dyspepsia, epigastric discomfort, weight loss, agitation, constipation, diarrhea, dry mouth, and bradycardia between the GBEs and the placebo group. Hu et al. (2013) reported that there was no difference in dizziness, headache, rash, insomnia, and diarrhea between the GBE and the placebo group.

\section{DISCUSSION}

\section{Summary of Evidence}

Ten systematic reviews were included in the present study. Eight out of the ten systematic reviews had high quality according to
OQAQ. To our knowledge, this is the first systematic review for systematic reviews on efficacy and safety of GBEs for MCI and dementia. Medication with GBEs showed improvement in cognition and daily activities, and the effect was dose-dependent. Significant efficacy was only demonstrated when they were used in high daily dose $(240 \mathrm{mg})$. Compared with placebo, adverse events were fewer in patients treated with GBE compared with placebo regarding the symptoms of dizziness/vertigo, tinnitus, headache, and angina pectoris. GBEs improve cognition functions but had no effect on ADL in MCI patients. However, there may be subtle impairment in instrumental ADLs in MCI patients, and then the scales used in earlier trials were certainly not sensitive enough to detect the presence or treatment-related changes in such subtle instrumental ADLs. There is no use measuring ADLs in patients with MCI or AAMI who have subtle or no ADL impairment, and then saying there was no improvement. GBEs improve NPS both in dementia patients and in the subgroup of $\mathrm{AD}$ patients. GBEs improve quality of life but couldn't change the progression of $\mathrm{AD}$ patients. However, the effects of GBEs on the progression of AD pathology have never been studied. Hence, it is not known if GBEs have such a diseasemodifying effect, but what has not been studied cannot just be denied.

\section{Limitations}

Some weaknesses exist in this study. (1) We also included reviews that did not clearly distinguish between Ginkgo extracts generated using different extraction methods. Extracts derived from the same plant can display substantial differences in composition, efficacy and safety. For instance, differential effects of EGb 761 have been demonstrated by Itil et al. (1996) when compared with other Ginkgo extracts. Efficacy of Ginkgo extracts was only demonstrated for the special extract EGb 761. (2) Even though most of these systematic reviews have high quality, the methodology of some primary trials may not be totally scientific which may result in some contradictory results. (3) By analyzing systematic reviews rather than clinical trials, important details of the primary studies may have been lost. (4) The weakness rested with primary studies. The objective of a systematic review is to provide a complete, exhaustive summary of current literature according to presetting a research question. The most important step is a thorough search of the relevant literature when conducting a systematic review. Thus, missing the important literature would undermine. In the present study, only a few systematic reviews included the large, recent trials by Ihl et al. (2011) and Herrschaft et al. (2012). Consequently, some reviews included here lack vital evidence and yielding inaccurate results.

\section{Implications for Future Research and Practice}

Our study provides a broad summary on all systematic reviews of GBEs for prevention, MCI and dementia which was essential for determining the efficacy of multipletherapeutic interventions, providing an important source for clinical and health policy decision making, as well as for clinical guidelines. GBEs are applied to the entire process of dementia, from prevention, 
MCI to dementia severe cases. In addition, GBEs seemed to be generally safe for clinical application.

Up to now, no drug has been demonstrated to prevent cognitive function declining and progression to $\mathrm{AD}$ in healthy people. However, in a 20 -year long follow-up populationbased study, Amieva et al. (2013) indicated that EGb 761 specifically prevented cognitive decline in a non-demented elderly population when compared with non-users. Thus, the long effects of GBEs on preventing cognitive function decline need to be further confirmed.

One systematic review focused on the MCI, indicating that there is mixed evidence to support efficacy of GBEs for MCI. A well designed RCT demonstrated that Ginkgo biloba extract EGb 761 improved cognitive functioning and aspects of quality of life in very mild MCI patients when compared with placebo control (Grass-Kapanke et al., 2011), and another one in MCI patients with neuropsychiatric symptoms (Gavrilova et al., 2014). However, no primary systematic review included this important RCT. Furthermore, a correct diagnosis is a crucial issue in every field lacking objective/instrumental markers of the disorder/syndrome under investigation (Halbreich, 2004). Based on the Diagnostic and Statistical Manual (DSM)-5 of the American Psychiatric Association, cognitive decline is divided into major and mild neurocognitive disorders (American Psychiatric Association, 2013). The DSM-5 classification has the advantage of covering the full range of MCI and dementia on the one hand and the different etiologic types of neurocognitive disorders on the other. However, the DSM-5 is not the only classification and diagnostic system that is appropriate to diagnose and select patients for clinical trials. A recent paper by Hoerr and Zaudig (2016) shows that the patients enrolled in the dementia trials meet the DSM-5 diagnostic criteria for major neurocognitive disorders and the patients enrolled in the recent MCI trials (as well as some older trials) meet the DSM-5 criteria for mild neurocognitive disorders. Thus, at least for the special Ginkgo extract EGb $761^{\circledR}$, there is no need to do new trials in patients defined by the DSM-5. Further rigorous RCT on GBEs should be performed according to appropriate diagnostic criteria.

\section{REFERENCES}

Al Faleh, K., and Al-Omran, M. (2009). Reporting and methodologic quality of Cochrane Neonatal review group systematic reviews. BMC Pediatr. 9:38. doi: 10.1186/1471-2431-9-38

Altamura, C., Scrascia, F., Quattrocchi, C. C., Errante, Y., Gangemi, E., Curcio, G., et al. (2016). Regional MRI diffusion, white-matter hyperintensities, and cognitive function in Alzheimer's disease and vascular dementia. J. Clin. Neurol. 12, 201-208. doi: 10.3988/jen.2016.12.2.201

American Psychiatric Association (2013). Sleep-Wake Disorders. Diagnostic and Statistical Manual of Mental Disorders, 5th Edn., (DSM-5). Arlington, VA: American Psychiatric Association.

Amieva, H., Meillon, C., Helmer, C., Barberger-Gateau, P., and Dartigues, J. F. (2013). Ginkgo biloba extract and long-term cognitive decline: a 20-year followup population-basedstudy. PLOS ONE 8:e52755. doi: 10.1371/journal.pone. 0052755

Apostolova, L. G. (2016). Alzheimer disease. Continuum (Minneap. Minn) 22, 419-434. doi: 10.1212/con.0000000000000307
There is clear evidence to support efficacy of GBEs for dementia, and the effect is dose and age-dependent. Efficacy was only demonstrated when they were used at a high daily dose (240 $\mathrm{mg}$ ), and efficacy was only demonstrated for the extract EGb 761. We recommend that dementia patients with the above characters can choose EGb 761 as a treatment. Regarding prevention of dementia in healthy people, it should be remembered that a lack of scientific evidence does not necessarily mean that the treatment is ineffective (Kotsirilos, 2005). Whether healthy patients at risk or lower doses of GBE, or Ginkgo extracts other than EGb 761 are effective, still needs to be evaluated by rigorous clinical trials using the best available scientific standards. The patients should be divided into more subgroups according to different ages, GBEs dosages, and DSM-5 classification criteria of cognitive decline.

\section{CONCLUSION}

The interests of the public and the medical profession in the use of GBEs for MCI and dementia have grown considerably in recent years. Our analysis supports the efficacy of GBEs for MCI and dementia of both the Alzheimer type and the vascular type of dementia, and of mixed dementias. In addition, GBEs are generally safe.

\section{AUTHOR CONTRIBUTIONS}

HZ, YZ, LH, QZ, HW, GZ, and YL designed the study; HZ and YZ collected the data; $\mathrm{HZ}$ and $\mathrm{YZ}$ performed all analyses; HZ, $\mathrm{YZ}, \mathrm{GZ}$, and YL wrote the manuscript. All authors contributed to writing of this manuscript.

\section{ACKNOWLEDGMENTS}

This project is supported by grant of the National Natural Science Foundation of China (81573750/81473491/81173395/H2902), the Young and Middle-Aged University Discipline Leaders of Zhejiang Province, China (2013277); Zhejiang Provincial Program for the Cultivation of High-level Health talents (2015).

Aygün, D., and Güngör, I. L. (2015). Why is Alzheimer disease confused with other dementias? Turk. J. Med. Sci. 45, 1010-1014. doi: 10.3906/sag-1405-47

Bogolepova, A. N. (2015). A modern concept of mixed dementia. Zh. Nevrol. Psikhiatr. Im. S. S. Korsakova. 115, 120-126. doi: 10.17116/ jnevro201511551120-126

Brooker, D., La Fontaine, J., Evans, S., Bray, J., and Saad, K. (2014). Public health guidance to facilitate timely diagnosis of dementia: Alzheimer's Cooperative Valuation in Europe recommendations. Int. J. Geriatr. Psychiatry 29, 682-693. doi: $10.1002 / g p s .4066$

Budson, A. E., and Solomon, P. R. (2012). New criteria for Alzheimer disease and mild cognitive impairment: implications for the practicing clinician. Neurologist 18, 356-363. doi: 10.1097/NRL.0b013e31826a998d

Chen, Y. D., Zhang, J., Wang, Y., Yuan, J. L., and Hu, W. L. (2016). Efficacy of cholinesterase inhibitors in vascular dementia: an updated meta-analysis. Eur. Neurol. 75, 132-141. doi: 10.1159/000444253

Crook, T. H., Larrabee, G. J., and Youngjohn, J. R. (1990). Diagnosis and assessment of age-associated memory impairment. Clin. Neuropharmacol. 13(Suppl. 3), S81-S91. 
Damiani, G., Silvestrini, G., Trozzi, L., Maci, D., Iodice, L., and Ricciardi, W. (2014). Quality of dementia clinical guidelines and relevance to the care of older people with comorbidity: evidence from the literature. Clin. Interv. Aging 9, 1399-1407. doi: 10.2147/CIA.S65046

Deardorff, W. J., Shobassy, A., and Grossberg, G. T. (2015). Safety and clinical effects of EVP-6124 in subjects with Alzheimer's disease currently or previously receiving an acetylcholinesterase inhibitor medication. Expert Rev. Neurother. 15, 7-17. doi: 10.1586/14737175.2015.995639

DeFeudis, F. V., and Drieu, K. (2000). Ginkgo biloba extract (EGb 761) and CNS functions: basic studies and clinical applications. Curr. Drug Targets 1, 25-58. doi: 10.2174/1389450003349380

DeKosky, S. T., Williamson, J. D., Fitzpatrick, A. L., Kronmal, R. A., Ives, D. G., Saxton, J. A., et al. (2008). Ginkgo evaluation of memory (GEM) study investigators. Ginkgo biloba for prevention of dementia: a randomized controlled trial. JAMA 300, 2253-2262. doi: 10.1001/jama. 2008.683

Dubois, B., Feldman, H. H., Jacova, C., Hampel, H., Molinuevo, J. L., Blennow, K., et al. (2014). Advancing research diagnostic criteria for Alzheimer's disease: the IWG-2 criteria. Lancet Neurol. 13, 614-629. doi: 10.1016/S14744422(14)70090-0

Fehske, C. J., Leuner, K., and Müller, W. E. (2009). Ginkgo biloba extract (EGb761) influences monoaminergic neurotransmission via inhibition of NE uptake, but not MAO activity after chronic treatment. Pharmacol. Res. 60, 68-73. doi: 10. 1016/j.phrs.2009.02.012

Fernández-Blázquez, M. A., Ávila-Villanueva, M., Maestú, F., and Medina, M. (2016). Specific features of subjective cognitive decline predict faster conversion to mild cognitive impairment. J. Alzheimers Dis. 52, 271-281. doi: 10.3233/JAD150956

Fitzpatrick-Lewis, D., Warren, R., Ali, M. U., Sherifali, D., and Raina, P. (2015). Treatment for mild cognitive impairment: a systematic review and metaanalysis. CMAJ Open 3, E419-E227. doi: 10.9778/cmajo.20150057

Gauthier, S., and Schlaefke, S. (2014). Efficacy and tolerability of Ginkgo biloba extract EGb $761^{\circledR}$ in dementia: a systematic review and meta-analysis of randomized placebo-controlled trials. Clin. Interv. Aging 9, 2065-2077. doi: 10. 2147/CIA.S72728

Gavrilova, S. I., Preuss, U. W., Wong, J. W. M., Hoerr, R., Kaschel, R., Bachinskaya, N., et al. (2014). Efficacy and safety of Ginkgo biloba extract EGb $761^{\circledR}$ in mild cognitive impairment with neuropsychiatric symptoms: a randomized, placebo-controlled, double-blind, multi-center trial. Int. J. Geriatr. Psychiatry 29, 1087-1095. doi: 10.1002/gps.4103

Grass-Kapanke, B., Busmane, A., Lasmanis, A., Hoerr, R., and Kaschel, R. (2011). Effects of Ginkgo biloba special extract EGb 761 in Very Mild Cognitive Impairment (vMCI). Neurosci. Med. 2, 48-56. doi: 10.4236/nm.2011.21007

Halbreich, U. (2004). The diagnosis of premenstrual syndromes and premenstrual dysphoric disorder-clinical procedures and research perspectives. Gynecol. Endocrinol. 19, 320. doi: 10.1080/0951590400018215

Herrschaft, H., Nacu, A., Likhachev, S., Sholomov, I., Hoerr, R., and Schlaefke, S. (2012). Ginkgo biloba extract EGb 761 in dementia with neuropsychiatric features: a randomised, placebo-controlled trial to confirm the efficacy and safety of a daily dose of $240 \mathrm{mg}$. J. Psychiatr. Res. 46, 716-723. doi: 10.1016/j. jpsychires.2012.03.003

Hill, N. L., Mogle, J. M., Munoz, E., Wion, R., and Colancecco, E. M. (2015). Assessment of subjective cognitive impairment among older adults. J. Gerontol. Nurs. 41, 28-35; quiz 36-37. doi: 10.3928/00989134-20150309-01

Hoerr, R., and Zaudig, M. (2016). A retrospective classification of diagnoses in terms of DSM-5 for patients included in randomized controlled trials of Ginkgo biloba extract EGb 761 ${ }^{\circledR}$. Eur. Arch. Psychiatry Clin. Neurosci. 266, 249-259. doi: 10.1007/s00406-015-0632-y

Howieson, D. B. (2016). Cognitive decline in presymptomatic Alzheimer disease. JAMA Neurol. 73, 384-385. doi: 10.1001/jamaneurol.2015.4993

Hu, Q., Tu, X., Zhang, Y., Yang, W. Y., and Long, J. (2013). Systemic review of Ginkgo biloba extract for vascular dementia. Liaoning J. Tradit. Chin. Med. 4, 683-686.

Hugo, J., and Ganguli, M. (2014). Dementia and cognitive impairment: epidemiology, diagnosis, and treatment. Clin. Geriatr. Med. 30, 421-442. doi: 10.1016/j.cger.2014.04.001

Ihl, R., Bachinskaya, N., Korczyn, A. D., Vakhapova, V., Tribanek, M., Hoerr, R., et al. (2011). Efficacy and safety of a once-daily formulation of Ginkgo biloba extract EGb 761 in dementia with neuropsychiatric features: a randomized controlled trial. Int. J. Geriatr. Psychiatry. 26, 1186-1194. doi: 10.1002/gps.2662 Ihl, R., Bunevicius, R., Frölich, L., Winblad, B., Schneider, L. S., Dubois, B., et al. (2015). WFSBP task force on mental disorders in primary care; WFSBP task force on dementia. World Federation of Societies of Biological Psychiatry guidelines for the pharmacological treatment of dementias in primary care. Int. J. Psychiatry Clin. Pract. 19, 2-7. doi: 10.3109/13651501.2014.961931

Itil, T. M., Eralp, E., Tsambis, E., Itil, K. Z., and Stein, U. (1996). Central nervous system effects of Ginkgo biloba, a plant extract. Am. J. Ther. 3, 63-73. doi: 10. 1097/00045391-199601000-00009

Janssen, I. M., Sturtz, S., Skipka, G., Zentner, A., Velasco Garrido, M., and Busse, R. (2010). Ginkgo biloba in Alzheimer's disease: a systematic review. Wien. Med. Wochenschr. 160, 539-546. doi: 10.1007/s10354-010-0844-8

Jiang, L., Su, L., Cui, H., Ren, J., and Li, C. (2013). Ginkgo biloba extract for dementia: a systematic review. Shanghai Arch. Psychiatry 25, 10-21. doi: 10. 3969/j.issn.1002-0829.2013.01.005

Jørgensen, K., Hasselbalch, S. G., and Waldemar, G. (2016). The risk of dementia and cognitive decline can be reduced. Ugeskr. Laeger. 178:V11150887.

Kanowski, S., and Hoerr, R. (2003). Ginkgo biloba extract EGb $761^{\circledR}$ in dementia: intent-to-treat analyses of a 24-week, multi-center, double-blind, placebocontrolled, randomized trial. Pharmacopsychiatry 36, 297-303. doi: 10.1055/s2003-45117

Kasper, S. (2015). Phytopharmaceutical treatment of anxiety, depression, and dementia in the elderly: evidence from randomized, controlled clinical trials. Wien. Med. Wochenschr. 165, 217-228. doi: 10.1007/s10354-015-0360-y

Kennedy, S., and Sud, D. (2014). A guide to prescribing anti-dementia medication. Nurs. Times 110, 16-18.

Kim, H. J., Cha, J., Lee, J. M., Shin, J. S., Jung, N. Y., Kim, Y. J., et al. (2016). Distinctive resting state network disruptions among Alzheimer's Disease, subcortical vascular dementia, and mixed dementia patients. J. Alzheimers Dis. 50, 709-718. doi: 10.3233/JAD-150637

Kotsirilos, V. (2005). Complementary and alternative medicine. Part 2-evidence and implications for GPs. Aust. Fam. Physician 34, 689-691.

Maurer, K., Ihl, R., Dierks, T., and Frölich, L. (1997). Clinical efficacy of Ginkgo biloba special extract EGb 761 in dementia of the Alzheimer type. J. Psychiatr. Res. 31, 645-655. doi: 10.1016/S0022-3956(97)00022-8

Mazza, M., Capuano, A., Bria, P., and Mazza, S. (2006). Ginkgo biloba and donepezil: a comparison in the treatment of Alzheimer's dementia in a randomized placebo-controlled double-blind study. Eur. J. Neurol. 13, 981-985. doi: 10.1111/j.1468-1331.2006.01409.x

McCarney, R., Fisher, P., Iliffe, S., van Haselen, R., Griffin, M., van der Meulen, J., et al. (2008). Ginkgo biloba for mild to moderate dementia in a community setting: a pragmatic, randomised, parallel-group, double-blind, placebo-controlled trial. Int. J. Geriatr. Psychiatry 23, 1222-1230. doi: 10.1002/ gps. 2055

Mitchell, S. L. (2015). Advanced dementia. N. Engl. J. Med. 373, 1275-1277. doi: 10. 1056/NEJMc1509349

Montine, T. J., Koroshetz, W. J., Babcock, D., Dickson, D. W., Galpern, W. R., Glymour, M. M., et al. (2014). ADRD 2013 conference organizing committee. recommendations of the Alzheimer's disease-related dementias conference. Neurology 83, 851-860. doi: 10.1212/WNL.0000000000000733

Moore, A., Patterson, C., Lee, L., Vedel, I., and Bergman, H. (2014). Canadian consensus conference on the diagnosis and treatment of dementia. Fourth canadian consensus conference on the diagnosis and treatment of dementia: recommendations for family physicians. Can. Fam. Physician 60, 433-438.

Mormino, E. C., and Papp, K. V. (2016). Cognitive decline in preclinical stage 2 Alzheimer disease and implications for prevention trials. JAMA Neurol. 73, 640-642. doi: 10.1001/jamaneurol.2016.0281

Napryeyenko, O., Borzenko, I., and GINDEM-NP Study Group. (2007). Ginkgo biloba special extract in dementia with neuropsychiatric features. A randomised, placebo-controlled, double-blind clinical trial. Arzneimittelforschung 57, 4-11. doi: 10.1055/s-0031-1296579

Napryeyenko, O., Sonnik, G., and Tartakovsky, I. (2009). Efficacy and tolerability of Ginkgo biloba extract EGb $761^{\circledR}$ by type of dementia: analyses of a randomised controlled trial. J. Neurol. Sci. 283, 224-229. doi: 10.1016/j.jns.2009.02.353

Newport, D. J., Carpenter, L. L., McDonald, W. M., Potash, J. B., Tohen, M., and Nemeroff, C. B. (2015). APA council of research task force on novel biomarkers and treatments. Ketamine and other NMDA antagonists: early clinical trials 
and possible mechanisms in depression. Am. J. Psychiatry 172, 950-966. doi: 10. 1176/appi.ajp.2015.15040465

Ngo, J., and Holroyd-Leduc, J. M. (2015). Systematic review of recent dementia practice guidelines. Age Ageing 44, 25-33. doi: 10.1093/ageing/ afu143

Noel-Storr, A. H., McCleery, J. M., Richard, E., Ritchie, C. W., Flicker, L., Cullum, S. J., et al. (2014). Reporting standards for studies of diagnostic test accuracy in dementia: the STARDdem Initiative. Neurology 83, 364-373. doi: 10.1212/ WNL.0000000000000621

O'Brien, J. T., and Thomas, A. (2015). Vascular dementia. Lancet 386, 1698-1706. doi: 10.1016/S0140-6736(15)00463-8

Oken, B. S., Storzbach, D. M., and Kaye, J. A. (1998). The efficacy of Ginkgo biloba on cognitive function in Alzheimer disease. Arch. Neurol. 55, 1409-1415. doi: 10.1001/archneur.55.11.1409

Petersen, R. C. (2016). Mild cognitive impairment. Continuum (Minneap. Minn) 22, 404-418. doi: 10.1212/con.0000000000000313

Prince, M., Wimo, A., Guerchet, M., Gemma-Claire, A., Wu, Y. T., and Prina, M. (2015). Data from: World Alzheimer Report 2015: The Global Impact of Dementia. London: Alzheimer's Disease International (ADI). Available online at: http://www.alz.co.uk/research/world-report-2015

Rockwood, K., Mitnitski, A., Black, S. E., Richard, M., and Defoy, I., VASPECT study investigators. (2013). Cognitive change in donepezil treated patients with vascular or mixed dementia. Can. J. Neurol. Sci. 40, 564-571. doi: 10.1017/ S0317167100014670

Scherrer, B., Andrieu, S., Ousset, P. J., Berrut, G., Dartigues, J. F., Dubois, B., et al. (2015). Analysing time to event data in dementia prevention trials: the example of the guidage study of EGb761. J. Nutr. Health Aging 19, 1009-1011. doi: 10. 1007/s12603-015-0661-2

Schmidt, R., Hofer, E., Bouwman, F. H., Buerger, K., Cordonnier, C., Fladby, T., et al. (2015). EFNS-ENS/EAN Guideline on concomitant use of cholinesterase inhibitors and memantine in moderate to severe Alzheimer's disease. Eur. J. Neurol. 22, 889-898. doi: 10.1111/ene.12707

Schneider, L. S., Mangialasche, F., Andreasen, N., Feldman, H., Giacobini, E., Jones, R., et al. (2014). Clinical trials and late-stage drug development for Alzheimer's disease: an appraisal from 1984 to 2014. J. Intern. Med. 275, 251-283. doi: 10. 1111/joim.12191

Ströhle, A., and Rapp, M. A. (2016). Prevention of cognitive decline: a physical exercise perspective on brain health in the long run. J. Am. Med. Dir. Assoc. 17, 461-462. doi: 10.1016/j.jamda.2016.02.030

Tan, M. S., Yu, J. T., Tan, C. C., Wang, H. F., Meng, X. F., Wang, C., et al. (2015). Efficacy and adverse effects of Ginkgo biloba for cognitive impairment and dementia: a systematic review and meta-analysis. J. Alzheimers Dis. 43, 589-603. doi: 10.3233/JAD-140837

Tchantchou, F., Lacor, P. N., Cao, Z., Lao, L., Hou, Y., Cui, C., et al. (2009). Stimulation of neurogenesis and synaptogenesis by bilobalide and quercetin via common final pathway in hippocampal neurons. J. Alzheimers Dis. 18, 787-798. doi: 10.3233/JAD-2009-1189

Tchantchou, F., Xu, Y., Wu, Y., Christen, Y., and Luo, Y. (2007). EGb 761 enhances adult hippocampal neurogenesis and phosphorylation of CREB in transgenic mouse model of Alzheimer's disease. FASEB J. 21, 2400-2408. doi: 10.1096/fj. 06-7649com

Thomas, S. P. (2016). Counseling older adults about cognitive decline. Issues Ment. Health Nurs. 37, 135-136. doi: 10.3109/01612840.2016.1140545

Thung, K. H., Yap, P. T., Adeli-M, E., and Shen, D. (2015). Joint Diagnosis and Conversion Time Prediction of Progressive Mild Cognitive Impairment (pMCI) Using Low-Rank Subspace Clustering and Matrix Completion. Med. Image Comput. Comput. Assist. Interv. 9351, 527-534. doi: 10.1007/978-3-31924574-4_63

Tsivgoulis, G., Katsanos, A. H., Papageorgiou, S. G., Dardiotis, E., Voumvourakis, K., and Giannopoulos, S. (2014). The role of neurosonology in the diagnosis of vascular dementia. J. Alzheimers Dis. 42(Suppl. 3), S251-S257. doi: 10.3233/ JAD-132441

van Dongen, M., van Rossum, E., Kessels, A., Sielhorst, H., and Knipschild, P. (2003). Ginkgo for elderly people with dementia and age-associated memory impairment: a randomized clinical trial. J. Clin. Epidemiol. 56, 367-376. doi: 10. 1016/S0895-4356(03)00003-9

Vellas, B., Coley, N., Ousset, P. J., Berrut, G., Dartigues, J. F., Dubois, B., et al. (2012). Long-term use of standardised Ginkgo biloba extract for the prevention of Alzheimer's disease (GuidAge): a randomised placebo-controlled trial. Lancet Neurol. 11, 851-859. doi: 10.1016/S1474-4422(12)70206-5

Wang, B. S., Wang, H., Song, Y. Y., Qi, H., Rong, Z. X., Wang, B. S., et al. (2010). Effectiveness of standardized Ginkgo biloba extract on cognitive symptoms of dementia with a six-month treatment: a bivariate random effect meta-analysis. Pharmacopsychiatry 43, 86-91. doi: 10.1055/s-0029-1242817

Wang, D. C., Black, S. E., Zukotynski, K. A., and Zukotynski, K. A. (2016). Diagnosing dementia. CMAJ 188, 603. doi: 10.1503/cmaj.150508

Weinmann, S., Roll, S., Schwarzbach, C., Vauth, C., and Willich, S. N. (2010). Effects of Ginkgo biloba in dementia: systematic review and meta-analysis. BMC Geriatr. 10:14. doi: 10.1186/1471-2318-10-14

Weitbrecht, W. U., and Jansen, W. (1986). Primary degenerative dementia: therapy with Ginkgo biloba extract. Placebo-controlled double-blind and comparative study. Fortschr. Med. 104, 199-202.

Winblad, B., Palmer, K., Kivipelto, M., Jelic, V., Fratiglioni, L., Wahlund, L.-O., et al. (2004). Mild cognitive impairment-beyond controversies, towards a consensus: report of the international working group on mild cognitive impairment. J. Intern. Med. 256, 240-246. doi: 10.1111/j.1365-2796.2004. 01380.x

Wood, H. (2016). Alzheimer disease: meta-analysis finds high reversion rate from MCI to normal cognition. Nat. Rev. Neurol. 12:189. doi: 10.1038/nrneurol. 2016.29

Yáñez, M., and Viña, D. (2013). Dual inhibitors of monoamine oxidase and cholinesterase for the treatment of Alzheimer disease. Curr. Top. Med. Chem. 13, 1692-1706. doi: 10.2174/15680266113139990120

Yang, G., Wang, Y., Sun, J., Zhang, K., and Liu, J. (2016). Ginkgo biloba for mild cognitive impairment and Alzheimer's disease: a systematic review and metaanalysis of randomized controlled trials. Curr. Top. Med. Chem. 16, 520-528. doi: 10.2174/1568026615666150813143520

Yang, M., Xu, D. D., Zhang, Y., Liu, X., Hoeven, R., and Cho, W. C. (2014). A systematic review on natural medicines for the prevention and treatment of Alzheimer's disease with meta-analyses of intervention effect of ginkgo. Am. J. Chin. Med. 42, 505-521. doi: 10.1142/S0192415X14500335

Yang, Z., Li, W. J., Huang, T., Chen, J. M., and Zhang, X. (2011). Meta-analysis of Ginkgo biloba extract for the treatment of Alzheimer's disease. Neural Regen. Res. 6, 1125-1129. doi: 10.3969/j.issn.1673-5374.2011.15.001

Yoshitake, T., Yoshitake, S., and Kehr, J. (2010). The Ginkgo biloba extract EGb 761(R) and its main constituent flavonoids and ginkgolides increase extracellular dopamine levels in the rat prefrontal cortex. Br. J. Pharmacol. 159, 659-668. doi: 10.1111/j.1476-5381.2009.00580.x

Conflict of Interest Statement: The authors declare that the research was conducted in the absence of any commercial or financial relationships that could be construed as a potential conflict of interest.

Copyright (c) 2016 Zhang, Huang, Zhong, Zhou, Wang, Zheng and Lin. This is an open-access article distributed under the terms of the Creative Commons Attribution License (CC BY). The use, distribution or reproduction in other forums is permitted, provided the original author(s) or licensor are credited and that the original publication in this journal is cited, in accordance with accepted academic practice. No use, distribution or reproduction is permitted which does not comply with these terms. 